\title{
Polymorphisms in the sialic acid-binding immunoglobulin-like lectin-8 (Siglec-8) gene are associated with susceptibility to asthma
}

\author{
Pei-Song Gao ${ }^{\star, 1}$, Kenichi Shimizu ${ }^{2}$, Audrey V Grant ${ }^{1}$, Nicholas Rafaels ${ }^{1}$, Lin-Fu Zhou ${ }^{1,3}$, Sherry A Hudson ${ }^{1}$, \\ Satoshi Konno ${ }^{2}$, Nives Zimmermann ${ }^{4}$, Maria I Araujo ${ }^{5}$, Eduardo V Ponte ${ }^{6}$, Alvaro A Cruz ${ }^{6}$, \\ Masaharu Nishimura ${ }^{2}$, Song-Nan $\mathrm{Su}^{7}$, Nobuyuki Hizawa ${ }^{8}$, Terry H Beaty ${ }^{9}$, Rasika A Mathias ${ }^{1}$, \\ Marc E Rothenberg ${ }^{4}$, Kathleen C Barnes ${ }^{1}$ and Bruce S Bochner ${ }^{1}$
}

\begin{abstract}
Sialic acid-binding immunoglobulin-like lectin-8 (Siglec-8) promotes the apoptosis of eosinophils and inhibits Fc\&RI-dependent mediator release from mast cells. We investigated the genetic association between sequence variants in Siglec-8 and diagnosis of asthma, total levels of serum IgE (tlgE), and diagnosis of eosinophilic esophagitis (EE) in diverse populations. The effect of sequence variants on Siglec-8 glycan ligand-binding activity was also examined. Significant association with asthma was observed for SNP rs36498 (odds ratios (OR), 0.69, $P=8.8 \times 10^{-5}$ ) among African Americans and for SNP rs10409962 (Ser/Pro) in the Japanese population (OR, $0.69, P=0.019)$. Supporting this finding, we observed association between SNP rs 36498 and current asthma among Brazilian families $(P=0.013)$. Significant association with tlgE was observed for SNP rs6509541 among African Americans $(P=0.016)$, and replicated among the Brazilian families $(P=0.02)$. In contrast, no association was observed with EE in Caucasians. By using a synthetic polymer decorated with $6^{\prime}$-sulfo-sLex, a known Siglec-8 glycan ligand, we did not find any differences between the ligand-binding activity of HEK293 cells stably transfected with the rs 10409962 risk allele or the WT allele. However, our association results suggest that the Siglec 8 gene may be a susceptibility locus for asthma.
\end{abstract} European Journal of Human Genetics (2010) 18, 713-719; doi:10.1038/ejhg.2009.239; published online 20 January 2010

Keywords: sialic acid-binding immunoglobulin-like lectin-8 (Siglec8); polymorphisms; asthma; eosinophilic esophagitis

\section{INTRODUCTION}

We and others have recently identified sialic acid-binding immunoglobulin-like lectin-8 (Siglec-8), a member of the CD33 subfamily of Siglecs. ${ }^{1}$ Siglec- 8 is a type-I transmembrane protein expressed on human eosinophils, mast cells, and basophils. ${ }^{2,3}$ This transmembrane protein has an extracellular binding domain that recognizes specific complex carbohydrates (glycans such as $6^{\prime}$-sulfated sialyl Lewis $\mathrm{X}$ $\left(6^{\prime}\right.$-sulfo-sLe $\left.\left.{ }^{\mathrm{x}}\right)\right){ }^{4}$ and intracellular immunoreceptor tyrosine-based inhibition motifs (ITIMs) that facilitate receptor binding into inhibitory signals. We have reported that incubation of human eosinophils with specific Siglec-8 monoclonal antibodies (mAbs) causes rapid caspaseand ROS-dependent apoptosis ${ }^{5-7}$ This was further supported by studies using mouse Siglec-F, the closest functional paralog of human Siglec-8, showing that Siglec-F engagement on murine eosinophils by antibodies induces their apoptosis in vitro, and administration of Siglec-F antibodies to hyper-eosinophilic mice reduces circulating and tissue eosinophils. ${ }^{8,9}$ Furthermore, mice deficient in mouse Siglec-F have exaggerated allergic lung eosinophilic responses. ${ }^{10}$ These findings underscore the importance of Siglec-F/8 in eosinophil apoptosis and human eosinophil-mediated disorders like asthma.
Eosinophils are involved in defense against parasites, but are also known for their role as effector cells involved in the pathobiology of a number of allergic diseases, including asthma and eosinophilic esophagitis (EE). ${ }^{11-13}$ It is well-recognized that the total eosinophil count in induced sputum reflects asthmatic activity and is useful for adjusting steroid dosage and for early detection of exacerbations. ${ }^{14,15}$ The involvement of Siglec-8 in eosinophil apoptosis supports the hypothesis that Siglec-8 may have a role in eosinophil-mediated asthma, allergic inflammation, and EE. Thus, abnormalities in Siglec-8 function due to genetic variations could be associated with the pathogenesis of asthma and other eosinophil-associated diseases. Genetic linkage studies have previously identified chromosomal regions that contain genes involved in the development of asthma and related phenotypes, including 19q13.33-q13.41, where Siglec-8 is located. ${ }^{16-18}$

Here, we explore the role of genetic polymorphisms in the Siglec-8encoding gene in asthma and other eosinophil-associated diseases. We selected tagging SNPs and functional SNPs encompassing Siglec-8 and conducted genetic association studies of healthy controls and a population of African Americans selected for asthma. The findings were replicated in Brazilian families characterized for current asthma

\footnotetext{
${ }^{1}$ Division of Allergy and Clinical Immunology, Department of Medicine, Johns Hopkins University School of Medicine, Baltimore, MD, USA; ${ }^{2}$ First Department of Medicine, Hokkaido University School of Medicine, Sapporo, Japan; ${ }^{3}$ Department of Respiratory Medicine, The First Affiliated Hospital, Nanjing Medical University, Nanjing, China; ${ }^{4}$ Division of Allergy and Immunology, Department of Pediatrics, Cincinnati Children's Hospital Medical Center, Cincinnati, OH, USA; ${ }^{5}$ Servico de Imunologia, Hospital Universitario Professor Edgard Santos, Salvador, Brazil; ${ }^{6}$ ProAR - Federal University of Bahia School of Medicine, Salvador, Brazil; ${ }^{7}$ Department of Medical Research and Education, Taipei Veterans General Veterans, Taipei, Taiwan; ${ }^{8}$ Department of Pulmonary Medicine, Graduate School of Comprehensive Human Sciences, University of Tsukuba, Ibaraki, Japan; ${ }^{9}$ Department of Epidemiology, Johns Hopkins Bloomberg School of Public Health, Baltimore, MD, USA

*Correspondence: Dr Pei-Song Gao, Johns Hopkins Asthma \& Allergy Center, 5501 Hopkins Bayview Circle, Baltimore, MD 21224 USA

Tel: +1 410550 2124; Fax: +1 410550 2130; E-mail: pgao1@jhmi.edu
}

Received 15 May 2009; revised 9 December 2009; accepted 10 December 2009; published online 20 January 2010 
and Japanese asthmatic patients and healthy controls. To determine whether the genetic association was applicable to other allergic diseases, we tested for association between Siglec-8 SNPs and EE in the Caucasian population. The effect of the non-synonymous coding SNP rs10409962 on Siglec-8 glycan ligand-binding activity was also examined.

\section{MATERIALS AND METHODS}

\section{Study population and phenotypes}

African American population. GRAAD (Genomic Research on Asthma in the African Diaspora) comprises NIH-funded studies of asthma in pediatric and adult African American populations and one study of healthy African Americans. ${ }^{19} \mathrm{~A}$ diagnosis of asthma was based on both a reported history of asthma and a documented history of physician-diagnosed asthma. For each of the asthma studies, a standardized questionnaire based on either the American Thoracic Society ${ }^{20}$ or The International Study of Asthma \& Allergy in Childhood (ISAAC) standards was administrated by a clinical coordinator. All controls were recruited separately and had no history of asthma. In the casecontrol design, 464 asthma cases ( $45.5 \%$ male, aged $23.8 \pm 17.9$ years) and 471 control subjects ( $41.4 \%$ male, aged $35.2 \pm 16.5$ years) were included in the final analysis. Detailed clinical characteristics of these subjects are presented in Table 1. Total serum IgE levels were measured using the UniCap 250 system (Pharmacia and Upjohn, Kalamazoo, MI, USA) at the Johns Hopkins DACI Laboratory (www.hopkinsmedicine.org/allergy/daci). ${ }^{21}$

Brazilian population. A total of 822 individuals among 356 nuclear families (44.2\% male, mean age 27 years) from five communities in the Conde District of Bahia, Brazil, were enrolled in the study (Table 1). Detailed information on these subjects has been described previously. ${ }^{22}$ The ISAAC questionnaire was administered to determine the prevalence and risk factors associated with asthma and allergic diseases. According to international ISAAC standards, current asthma was defined as a combination of wheezing in the past 12 months and lifetime asthma. Stool samples of the subjects were examined by the Kato-Katz method ${ }^{23}$ for the presence and parasite load of Ascaris lumbricoides, Trichuris trichiura, and Hookworm eggs. Total serum levels of IgE were measured by chemiluminescence (ADVIA Center Bayer Corporation, Tarrytown, NY, USA). Detailed demographic and phenotype summary data of these individuals are presented in Table 1.

Japanese population. A total of 468 subjects with asthma (male, $47.0 \%$, aged $45.9 \pm 16.8$ years) and 457 healthy control subjects (male, 64.6\%, aged $38.8 \pm 13.6$ years) were recruited from the pulmonary clinic at the First Department of Medicine, Hokkaido University Hospital, as described previously. ${ }^{24}$ The criteria for asthma in the Japanese population are presented in the Online Supplementary Information, and detailed clinical characteristics of these subjects are presented in Table 1.
Caucasians with EE. EE patients were recruited through the Cincinnati Center for Eosinophilic Disorders. EE was defined as 24 eosinophils/h.p.f. in at least one high-power field $(\times 400)$ of an esophageal biopsy as previously described. ${ }^{25}$ As shown in Table 1, a total of 298 Caucasians were included for the final analyses, including $166 \mathrm{EE}$ patients from the proband of each family and 132 race/ethnicity-matched unrelated healthy individuals. DNA was isolated from saliva, peripheral blood leukocytes, or buccal swab samples.

The study protocols were approved by the Institutional Review Boards at the Johns Hopkins University School of Medicine, Cincinnati Children's Hospital Medical Center, the Federal University of Bahia, Brazil, and by the Ethics Committee, Hokkaido University, Japan. Informed consent was obtained from each of the study subjects.

\section{Genotyping and quality control}

A total of 14 SNPs were genotyped in the African American subjects from GRAAD. These SNPs included seven SNPs in the Illumina HumanHap650Y panel, additional tagging SNPs for the African American population encompassing Siglec-8, and two coding SNPs (rs3829659 Arg388Gly and rs10409962 Ser170Pro). Details for each SNP and minor-allele frequencies (MAFs) are presented in Supplementary Table S1, and the strategy for SNP selection is described in the Online Supplementary Information. Seven SNPs included in Illumina HumanHap650Y version 1 and 3 BeadChips (rs5409541, rs10518263, rs39711, rs10408249, rs36496, rs36498, rs11672925) were genotyped using the Illumina's BeadArray at the Center for Inherited Disease Research (CIDR) as described previously. ${ }^{19}$ The rest of the SNPs were genotyped using customdesigned primers and probes for the TaqMan Allelic Discrimination assays using the 7900HT Sequence Detection System (Applied Biosystems, Foster City, CA, USA). ${ }^{26}$

Six SNPs, including four SNPs in the regulatory regions (rs6509541, rs39711, rs10420357, rs36498) and two (rs3829659 Arg388Gly and rs10409962 Ser170Pro) in the coding regions of the Siglec- 8 gene, were genotyped among 356 nuclear Brazilian families using TaqMan assays. Additionally, findings from both African American case-control and Brazilian family-based studies (rs36498 and rs10409962), together with the additional coding SNP rs3829659, were replicated in the Japanese population. Among them, the SNPs rs10409962 and rs3829659 were genotyped using TaqMan assay, and SNP rs36498 was genotyped using an assay combining kinetic PCR with allelespecific amplification, as described previously. ${ }^{27}$ Additionally, eight SNPs, including those associated with asthma, coding SNPs, and tagging SNPs across the Siglec- 8 gene for the Caucasians from the International HapMap project, were genotyped in a total of 298 Caucasians consisting of 166 EE patients and 132 controls using TaqMan assays. Quality control for genotyping was described previously. ${ }^{19,28}$ Briefly, quality-control measures included a genotyping call rate of $>95 \%$, with no discordances on repeat genotyping of

Table 1 Clinical characteristics of the study populations

\begin{tabular}{|c|c|c|c|c|c|c|}
\hline \multirow[b]{2}{*}{ Panel $A$} & \multirow[b]{2}{*}{ Total } & \multicolumn{3}{|c|}{ African American } & \multicolumn{2}{|c|}{ Japanese } \\
\hline & & Asthma & Controls & Total & Asthma & Controls \\
\hline Subjects $(n)$ & 935 & 464 & 471 & 925 & 468 & 457 \\
\hline Males, $n(\%)$ & $406(43.4 \%)$ & $211(45.5 \%)$ & $195(41.4 \%)$ & $515(55.7 \%)$ & $220(47.0 \%)$ & 295 (64.6\%) \\
\hline Age; mean (SD) & $29.55(18.10)$ & $23.78(17.85)$ & $35.23(16.51)$ & $42.4(15.7)$ & $45.9(16.8)$ & $38.8(13.6)$ \\
\hline Total IgE; mean ${ }^{\mathrm{a}}(95 \% \mathrm{Cl})$ & $213.7(191.5-238.4)$ & $315.6(270.4-368.3)$ & $143.3(123.8-165.8)$ & $132.6(119.6-147.0)$ & $242.7(211.0-279.1)$ & $71.8(63.1-81.7)$ \\
\hline \multirow[t]{2}{*}{ Atopy, $n(\%)$} & $641(75.2 \%)$ & 369 (85.2\%) & 272 (64.9\%) & 587 (63.5\%) & 339 (72.4) & $248(54.3)$ \\
\hline & & & Brazilian families & & \multicolumn{2}{|c|}{ Caucasian } \\
\hline Panel B & Total & Wheeze & Control & Total & $\mathrm{EE}$ & Control \\
\hline Subjects $(n)$ & 822 & 244 & 550 & 298 & 166 & 132 \\
\hline Males (\%) & $363(44.2 \%)$ & $114(46.7 \%)$ & $233(42.4 \%)$ & $166(55.7 \%)$ & $102(61.4 \%)$ & $64(48.5 \%)$ \\
\hline Age; mean (SD) & $26.9(18.75)$ & $26.6(18.87)$ & $27.0(18.70)$ & - & - & - \\
\hline Total IgE; mean ${ }^{a}(95 \% \mathrm{Cl})$ & $2505.6(2296.1-2734.3)$ & $2631.2(2292.9-3019.5)$ & $2450.9(2194.3-2737.4)$ & - & - & - \\
\hline
\end{tabular}

aGeometric mean serum levels of total IgE. 
a random $5 \%$ of the sample, no significant departures from Hardy-Weinberg Equilibrium within each study group (exact $P$-value $>0.01$ ), $\operatorname{MAF}>5 \%$, and Mendelian errors in 5\% or more of the marker. Population admixture among African Americans was evaluated by genotyping 416 SNPs identified as ancestry-informative markers (AIMs) as described previously. ${ }^{19}$

\section{Plasmid constructs}

The wild-type (WT) 246 (pFlag-Neo-Siglec-8L) construct containing fulllength Siglec-8 was provided by Dr Oksoon H Choi (Johns Hopkins Asthma \& Allergy Center, Baltimore, MD, USA). The plasmid construct containing the SNP rs10409962-mutant allele was generated using the QuikChange XL sitedirected mutagenesis kit (Stratagene, La Jolla, CA, USA), by making a point mutation at 1160 from $\mathrm{T}$ to $\mathrm{C}$ in the double-stranded DNA from 246(pFlagNeo-Siglec-8L) (Figure 2a). The mutated plasmid was confirmed by direct sequencing using an ABI 3700 DNA Analyzer.

\section{Generation of stable Siglec-8 transfectants and Siglec-8 ligand binding assays}

HEK293 human embryonic kidney cells were cultured in MEM (Invitrogen, Carlsbad, CA, USA) supplemented with $10 \%$ fetal bovine serum (High Clone Laboratories, Logan, UT, USA), and $100 \mathrm{U} / \mathrm{ml}$ penicillin-G. The plasmid DNA $(1 \mu \mathrm{g})$ coding for WT or rs10409962-mutant Siglec-8 was transfected into HEK293 cells using the FuGENE-6 reagent (Roche, Indianapolis, IN, USA). The cells were harvested $24 \mathrm{~h}$ after transfection. Siglec- $8 \mathrm{mAb} 2 \mathrm{C} 4$ (mouse $\mathrm{IgG1})^{2}$ was used to detect cell-surface expression and a $2000-\mathrm{kDa}$ polyacrylamide biotinylated polymer decorated with $6^{\prime}$-sulfo-sLe ${ }^{\mathrm{x}}$ (provided by $\mathrm{Dr} \mathrm{N}$ Bovin, Russian Academy of Sciences, Moscow, Russia) was used in flow cytometric ligand binding assays as previously described. ${ }^{29}$ Briefly, cultured cells were washed once in PBS and incubated with either mAb 2C4 or an IgG1 isotype control antibody $\left(2.5 \mu \mathrm{g} / \mathrm{ml}, 30 \mathrm{~min}, 4^{\circ} \mathrm{C}\right)$, washed, and then incubated with a fluorochrome-conjugated anti-mouse $\operatorname{IgG} \mathrm{F}\left(\mathrm{ab}^{\prime}\right) 2$ secondary antibody (Invitrogen, CA, USA). Alternatively, cells were incubated with saturating concentrations $(10 \mu \mathrm{g} / \mathrm{ml})$ of biotinylated a $2000-\mathrm{kDa} 6^{\prime}$-sulfo-sLe ${ }^{\mathrm{x}}$-containing polyacrylamide polymer or a control $2000-\mathrm{kDa}$ LacNac polymer, ${ }^{29}$ washed, and then incubated with saturating concentrations of FITC-strepavidin (R\&D Systems, Minneapolis, MN, USA). The cells were then analyzed by flow cytometry (FACS Calibur; Becton-Dickinson, San Jose, CA, USA).

\section{Statistical analysis}

The Cochran-Armitage trend test was used to examine associations between each individual marker (under an additive model) and disease status among African Americans and Caucasians using the PLINK software (http:// pngu.mgh.harford.edu/ purcell/plink/to). ${ }^{30}$ We tested for associations between SNPs and total serum levels of log-transformed IgE by a linear regression analysis under an additive model adjusted for confounding variables (age and gender). Departures from Hardy-Weinberg equilibrium at each locus were tested by $\chi^{2}$-test separately for cases and controls, and for founders among Brazilian families. Haplotype analyses were performed with PLINK, using sliding windows of 2-5 SNPs, where empiric $P$-values for haplotype frequency differences were generated over 10000 permutations. For the Brazilian familybased analyses, Mendelian inconsistencies were identified with Sib-Pair (version $1.00 \mathrm{~b})$. Association between markers in Siglec- 8 and wheezing, $\log [\operatorname{tgEE}]$, and $\log [$ Schistosoma mansoni egg count] were tested by using FBAT (version 1.5.5). ${ }^{31}$ All analyses with FBAT were first performed under an additive and then by a recessive model. Statistical analysis of the Japanese population used logistic regression analysis to estimate the odds ratio (OR) for asthma using an additive model adjusted and un-adjusted for gender, age, and smoking status using SYSTAT (version 10.2; Systat Inc., Chicago, IL, USA). ${ }^{24}$ Statistical power for the case-control study was estimated using QUANTO (version 1.2) $125 .^{32}$

\section{RESULTS}

Clinical features, SNP information, and the LD structure

Clinical characteristics of the study subjects are presented in Table 1 . The African American population is the primary focus and study population; both Brazilian and Japanese populations were used for replication of any significant findings in the primary data analysis. The chromosomal location and MAFs for the 14 Siglec-8 SNPs spanning a $16-\mathrm{kb}$ region on chromosome $19 \mathrm{q} 13.33-\mathrm{q} 13.41$ for healthy controls from our study populations (African American, Brazilian, Japanese, Caucasian) and from the International HapMap project (http:// www.hapmap.org/) are presented in Supplementary Table S1. The genotype frequencies for all SNPs agreed with expectations under Hardy-Weinberg equilibrium. Power calculation for each population is presented in Supplementary Figure S1.

\section{Association between Siglec-8 variants and asthma and total serum} levels of IgE (tIgE)

We observed two linkage disequilibrium (LD) blocks, comprising five markers in block-1 (rs36485, rs36487, rs36489, rs10518263, rs39711) and two markers in block-2 (rs3829659 and rs10408249), for Siglec-8 among African Americans following the definition of Gabriel et al..$^{33}$ (Supplementary Figure S2a). In primary analysis, we observed an association for the promoter SNP rs36498 $(-4354 \mathrm{C} / \mathrm{T})$, where the $\mathrm{T}$ allele was significantly associated with reduced risk of asthma (OR, 0.69, 95\% CI, 0.57-0.83, $P=8.8 \times 10^{-5}$; Table 2). A similar trend toward reduced risk of asthma was also seen for a coding SNP

Table 2 Allelic association of Siglec-8 SNPs with asthma in both African American and Japanese ( $n=957)$ populations

\begin{tabular}{|c|c|c|c|c|c|c|c|c|c|}
\hline \multirow[b]{2}{*}{ SNP } & \multirow[b]{2}{*}{ Minor allele } & \multicolumn{4}{|c|}{ African American $(n=935)$} & \multicolumn{4}{|c|}{ Japanese $(n=957)$} \\
\hline & & Asthma & Control & OR $(95 \% \mathrm{Cl})$ & $P$ & Asthma & Control & OR $(95 \% \mathrm{Cl})$ & $\mathrm{Pa}$ \\
\hline rS11672925 & C & 0.220 & 0.194 & $1.17(0.92-1.48)$ & 0.195 & & & & \\
\hline rs36498 & $\mathrm{T}$ & 0.406 & 0.499 & $0.69(0.57-0.83)$ & $8.8 \times 10^{-5}$ & 0.340 & 0.330 & $1.01(0.83-1.24)$ & 0.899 \\
\hline rs10420357 & $\mathrm{T}$ & 0.269 & 0.250 & $1.10(0.87-1.40)$ & 0.414 & & & & \\
\hline rs36496 & C & 0.048 & 0.043 & $1.13(0.71-1.81)$ & 0.594 & & & & \\
\hline rs10409962 & G (Ser/Pro) & 0.446 & 0.497 & $0.82(0.65-1.02)$ & 0.070 & 0.100 & 0.132 & $0.69(0.50-0.94)$ & 0.019 \\
\hline rs36495 & $A$ & 0.376 & 0.361 & $1.04(0.85-1.27)$ & 0.549 & & & & \\
\hline rs10408249 & C & 0.482 & 0.469 & $1.05(0.87-1.27)$ & 0.571 & & & & \\
\hline rs3829659 & $\mathrm{G}(\mathrm{Arg} / \mathrm{G} \ln )$ & 0.484 & 0.457 & $1.12(0.89-1.40)$ & 0.321 & 0.327 & 0.360 & $0.93(0.75-1.15)$ & 0.515 \\
\hline rs39711 & $\mathrm{T}$ & 0.478 & 0.448 & $1.13(0.93-1.36)$ & 0.216 & & & & \\
\hline rs10518263 & $\mathrm{T}$ & 0.100 & 0.116 & $0.84(0.62-1.15)$ & 0.273 & & & & \\
\hline rs36489 & G & 0.151 & 0.167 & $0.89(0.67-1.19)$ & 0.410 & & & & \\
\hline rs36487 & $A$ & 0.135 & 0.149 & $0.89(0.66-1.19)$ & 0.423 & & & & \\
\hline rs36485 & $\mathrm{T}$ & 0.325 & 0.292 & $1.17(0.93-1.47)$ & 0.171 & & & & \\
\hline rs6509541 & $\mathrm{T}$ & 0.499 & 0.498 & $1.01(0.83-1.22)$ & 0.926 & & & & \\
\hline
\end{tabular}

aAdjusted for age, sex, smoking status, and atopic status. Bold $P$-values are statistically significant. 


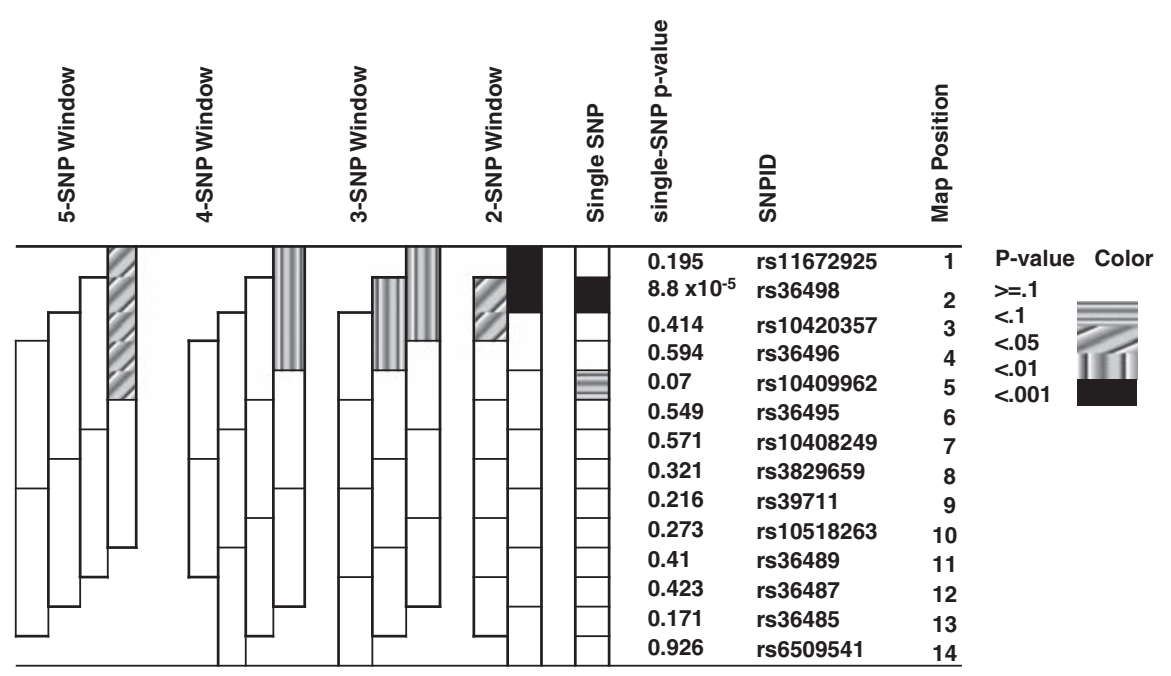

Figure 1 Summary of sliding-window analysis of Siglec-8 haplotypes and asthma in the African American population. Haplotype results show global $P$-values constructed across sliding windows of sizes $2-5$ for 14 SNPs and asthma. The color key for $P$-values is also indicated.

Table 3 Association of the two-SNP haplotype (rs36498 and rs11672925) in the promoter region of Siglec- 8 and asthma among African Americans

\begin{tabular}{llcccc}
\hline \multicolumn{6}{c}{ Minor allele frequencies } \\
rs36498 & rs11672925 & Asthma & Control & OR $(95 \% \mathrm{Cl})$ & P-value \\
\hline $\mathrm{T}$ & $\mathrm{C}$ & 0.105 & 0.097 & $1.09(0.70-1.71)$ & 0.576 \\
$\mathrm{C}$ & $\mathrm{C}$ & 0.112 & 0.094 & $1.22(0.78-1.92)$ & 0.191 \\
$\mathrm{~T}$ & $\mathrm{~A}$ & 0.304 & 0.401 & $0.65(0.49-0.86)$ & $\mathbf{1 . 4 2 \times 1 0 ^ { - 5 }}$ \\
$\mathrm{C}$ & $\mathrm{A}$ & 0.479 & 0.408 & $1.33(1.02-1.74)$ & $\mathbf{0 . 0 0 2}$ \\
\hline
\end{tabular}

Global $P$-value $=0.00026$. Bold $P$-values are statistically significant.

Table 4 Association of Siglec-8 haplotypes with total serum IgE among the African Americans $(n=935)$

\begin{tabular}{lccccccc} 
Single-SNP & rs6509541 & $r s 36485$ & $r s 36487$ & $r s 36489$ & $r s 10518263$ & & \\
Risk allele & $T$ & $C$ & $C$ & $A$ & $G$ & & \\
$P$-value & 0.016 & 0.961 & 0.896 & 0.935 & 0.476 & $\beta$ & $P$ \\
\hline Two-SNP & $\mathrm{T}$ & $\mathrm{C}$ & & & & -0.07 & 0.122 \\
Three-SNP & $\mathrm{T}$ & $\mathrm{C}$ & $\mathrm{C}$ & & & -0.271 & 0.004 \\
Four-SNP & $\mathrm{T}$ & $\mathrm{C}$ & $\mathrm{C}$ & $\mathrm{A}$ & & -0.275 & 0.005 \\
Five-SNP & $\mathrm{T}$ & $\mathrm{C}$ & $\mathrm{C}$ & $\mathrm{A}$ & $\mathrm{G}$ & -0.254 & $\mathbf{0 . 0 1}$ \\
\hline
\end{tabular}

$\beta$, regression coefficient. Bold $P$-values are statistically significant.

rs10409962 on exon-2, which causes a Ser-Pro substitution at amino acid 170 (S170P) in domain-2 (OR, 0.82, 95\% CI, 0.65-1.02, $P=0.072$ ). Sliding-window haplotype analysis across all 14 SNPs revealed significant associations for the 2-5 SNP haplotypes and risk of asthma (global P-values, 0.008-0.00026) (Figure 1), all of which included SNP rs36498, with the strongest association for a two-SNP haplotype (TA) comprising two promoter SNPs of Siglec-8 (rs36498 and rs11672925) (asthma versus control, 30.4\% versus $40.1 \%$; OR, $0.65 ; P=1.42 \times 10^{-5}$; global $P$-value $=0.00026$ ) (Table 3 ), suggesting enhanced association for the haplotype analysis in the promoter region of Siglec-8.
To determine whether Siglec-8 SNPs are associated with tIgE, we performed linear regression analysis using additive models adjusted for confounding variables (age and gender). Among all tested SNPs, SNP rs6509541 in the $3^{\prime}$-untranslated region (3'-UTR) showed significant association with $\operatorname{tgE}(P=0.016)$ (Table 4$)$. This association was further enhanced by haplotype analysis: multiple haplotypes of 2-5 SNPs were significantly associated with $\operatorname{tgE}$ ( $P$-values, $0.048-0.004)$ in the African American population, all of which included SNP rs6509541, with the strongest association for a three-SNP haplotype (TCC) consisting of SNPs in the $3^{\prime}$-UTR and downstream from Siglec-8 (rs6509541, rs36485, rs36487; $P=0.004$ ).

Replication of the primary findings in both Brazilian and Japanese populations

To further confirm these findings, we selected both rs36498 and rs10409962 SNPs that were associated with asthma among the African American subjects and five additional SNPs in the regulatory regions of Siglec-8 with even distribution and performed a family-based association study of 356 Brazilian nuclear families..$^{22}$ Detailed location and allele frequencies for all the genotyped SNPs among the Brazilian families are presented in the Supplementary Table S1. Of interest, significant evidence of linkage to current asthma was provided for rs36498 $(P=0.013)$ and suggestive evidence was found for the marker rs10409962 $(P=0.085)$ under the recessive model (Supplementary Table S2). In addition, association with tIgE was also observed for SNP rs6509541 among the Brazilian families $(P=0.02$, data not shown), which was the same SNP downstream from Siglec-8 that was associated with $\operatorname{tg} E$ in the African Americans. Haplotype analyses did not provide any further evidence for this association for either current asthma or tIgE. Given the association of rs36498 and rs10409962 with asthma in both African American and Brazilian subjects, we further replicated two associated SNPs rs36498 and rs10409962 and one additional coding SNP rs3829659 in the Japanese population comprising 468 asthmatics and 457 healthy control subjects. The association with reduced risk of asthma was replicated for rs10409962 (OR, 0.73; 95\% CI, 0.54-0.98; $P=0.032$ ) but not for SNP rs36498 (OR, 1.04; 95\% CI, 0.86-1.27; $P=0.67)$ and rs3829659 (OR, 0.86; 95\% CI, 0.71-0.98; $P=0.088$ ) (data not shown). As there 


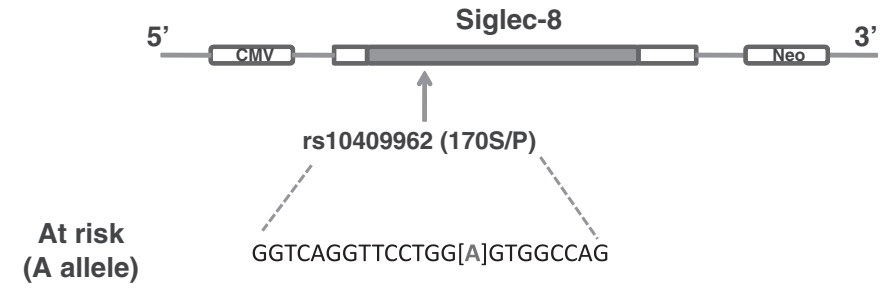

Protective

(G allele)

GGTCAGGTTCCTGG[G]GTGGCCAG

b
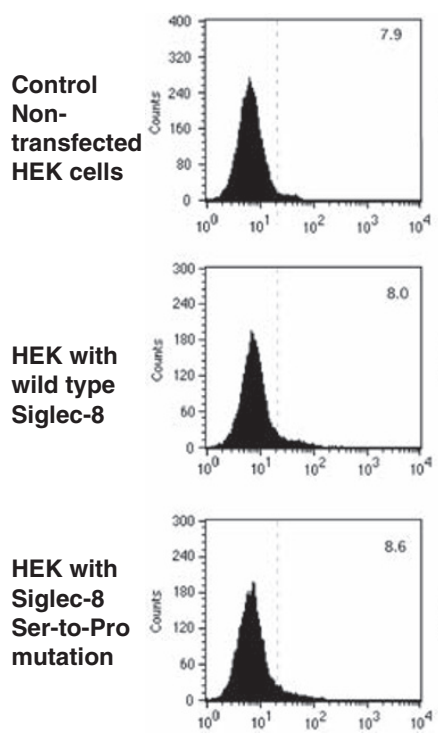

Siglec-8
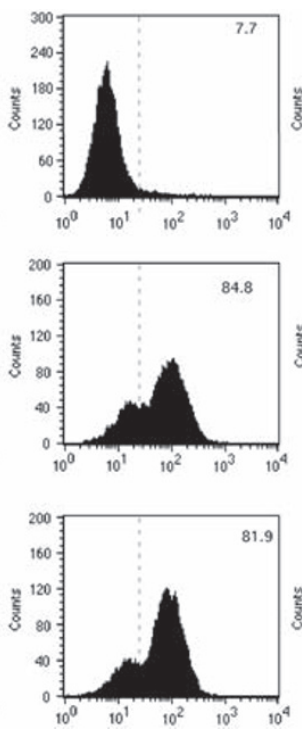

Control polymer
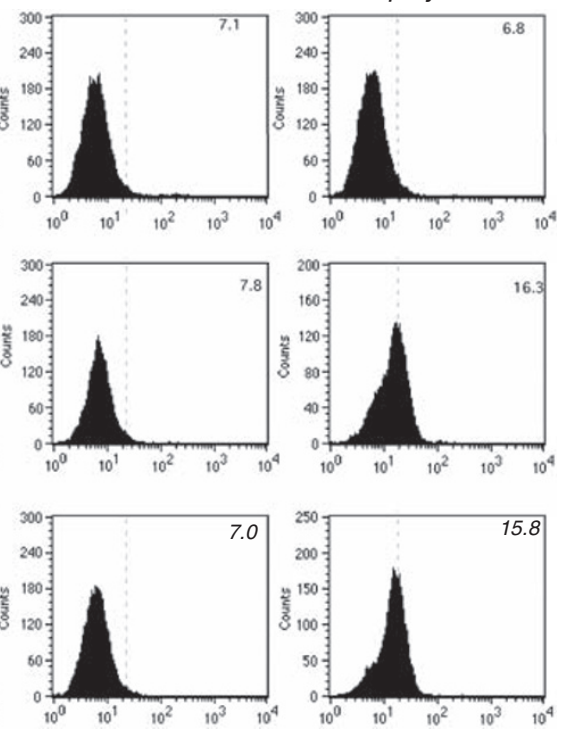

Siglec-8 ligand polymer
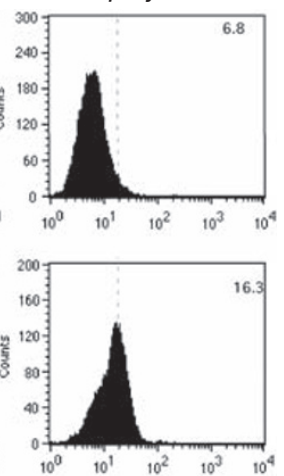

Fluorescence intensity

Figure 2 The pFlag-Neo-Siglec-8 plasmid construct and Siglec-8 expression on Siglec-8-transfected HEK293 cells. (a) The pFlag-Neo-Siglec-8 plasmid constructs contains either the SNP rs10409962 risk (A allele) or protective allele (G allele). (b) Detection of Siglec-8 expression on Siglec-8-transfected HEK293 cells by means of flow cytometry. Cells were analyzed for specific staining with isotype (mouse IgG), Siglec-8 antibody (2C4), and Siglec-8 ligand (6'-sulfo-sLex polymer) in HEK293 cells non-transfected (top), transfected with Siglec-8 WT (middle), and transfected with the Siglec-8 Ser-to-Pro mutation (bottom). The data represent one of three experiments. Values in the upper right corner of each histogram represent percent positive.

were significant differences in male/female, age distribution, atopy, and smoking status between the Japanese cases and controls $(P<0.001$ for all comparisons), ${ }^{24}$ further analysis was performed by adjusting for age, sex, smoking, and atopic status, and we found that the association for SNP rs10409962 still remained significant (OR, 0.69; 95\% CI, $0.50-0.94 ; P=0.019 ;$ Table 2). None of the Siglec-8 SNPs were associated with $\operatorname{tgE}$ in the Japanese population (data not shown).

\section{No association between Siglec-8 SNPs and EE}

Given the biological relationship between Siglec-8 and eosinophil apoptosis, we tested for association between eight Siglec-8 SNPs and EE, a disorder frequently associated with atopy and characterized by aberrant and localized accumulation of eosinophils in the esophagus. ${ }^{34}$ These SNPs, including those associated with asthma, were genotyped using TaqMan assays. We observed two LD blocks comprising six SNPs in block-1 (rs36485, rs36487, rs36489, rs39711, rs3829659, rs36495) and two SNPs in block-2 (rs10409962 and rs10420357) (Supplementary Figure S2B). Genotype frequencies for all SNPs were in HardyWeinberg equilibrium in both EE patients and control individuals without EE. However, none of the SNPs were significantly associated with EE, even after adjusting for gender (Supplementary Table S3).
HEK293 cells carrying a Ser-Pro substitution in exon-2 at position 170 of Siglec-8 exhibit normal ligand-binding activity

The coding SNP rs10409962 is located not far from the putative carbohydrate-binding domain of Siglec-8. To investigate whether SNP rs10409962 can lead to a functional change in Siglec-8, we examined the glycan ligand-binding activity of Siglec-8. HEK cells were stably transfected with either WT or the mutant allele, and selected for clones with surface levels of Siglec-8 similar to that on eosinophils (Figure $2 \mathrm{~b}$ ). When tested for their ability to bind the $2000-\mathrm{kDa} 6^{\prime}$ sulfo-sLe ${ }^{\mathrm{x}}$-containing polyacrylamide polymer ligand, there was no difference in ligand-binding activity between cells transfected with SNP rs10409962 risk allele and those transfected with the WT allele ( 84.8 versus $81.9 \%$ positive), suggesting that this point mutation does not significantly alter the ligand-binding activity of Siglec- 8 .

\section{DISCUSSION}

Siglec- 8 is expressed exclusively on human eosinophils, mast cells, and basophils. ${ }^{2}$ It has been demonstrated that Siglec- 8 engagement induces eosinophil apoptosis in vitro by triggering the 'intrinsic' stressmediated apoptotic pathway through sequential ROS production, mitochondrial dysfunction, and caspase cleavage. ${ }^{3}$ Of interest, murine 
studies targeting Siglec-F, the closest functional paralog to Siglec-8, also demonstrated a significant role in regulating the pathogenesis of eosinophil-mediated disorders. ${ }^{8-10}$ As eosinophils and their many biologically active mediators are associated with allergic diseases and other chronic inflammatory disorders, this suggests that Siglec-8 could be a candidate gene for human eosinophilic disorders like asthma and EE. In this study, we tested the hypotheses that genetic polymorphisms in Siglec- 8 are associated with asthma and EE, and that mutations in the glycan-binding extracellular domain of Siglec- 8 could disrupt the affinity of Siglec-8 for its ligand, which might then lead to loss of ligand-induced apoptosis in vivo, resulting in exaggerated eosinophilic inflammation.

We took advantage of genome-wide association studies (GWAS) originally performed to identify susceptibility genes for asthma in a total of 935 African Americans. ${ }^{19}$ Seven Siglec-8 SNPs were included in the GWAS final data analysis after all the filtering processes were performed for quality control. In parallel with this analysis, an additional seven SNPs were genotyped. Of these, two common nonsynonymous variants (rs3829659 Arg388Gly and rs10409962 Ser170Pro) were located along the functional regions of the Siglec- 8 gene in the glycan-binding extracellular domain (Ser170Pro) and the cytoplasmic negative signaling domain (Arg388Gly). Among all the SNPs genotyped, the strongest evidence for association with asthma was observed for the promoter SNP rs36498 (-4354C/T), and suggestive evidence for the non-synonymous coding SNP rs10409962 (Ser170Pro) on exon-2, where both mutant alleles were associated with reduced risk of asthma. When replicating these findings in two additional independent populations, similar associations were found, namely SNP rs36498 was significantly, and rs10409962 was suggestively, associated with current asthma as documented by a combination of wheezing in the past 12 months and lifetime asthma among Brazilian families. In contrast, SNP rs10409962, but not rs36498, was significantly associated with asthma in the Japanese case-control analysis. These findings suggest that genetic variants in the Siglec- 8 gene may be associated with asthma. Although we did not observe SNP-for-SNP replication of findings in these populations, this was not unexpected. As highlighted in Supplementary Table S1, the allele frequencies of all the SNPs genotyped differed across ethnic groups, suggesting there was considerable genetic heterogeneity among these populations. It is possible that variants other than those tested here are causal and directly alter Siglec-8's function, and the observed statistical associations in this study are merely due to strong LD between markers and these unobserved causal variants. Heterogeneity of the phenotype is another commonly cited explanation for failure to replicate positive associations across independent populations. However, it is important to note that SNPs in each population showed associations with asthma, providing a convincing basis for further investigation of the role of Siglec-8 in the pathogenesis of asthma. In addition, although SNP rs10409962 failed to pass the correction for Bonferroni Multiple testing, the most stringent test of all, the associated SNP, rs10409962, is located two amino acids away from a putative carbohydrate-binding domain. It is still possible that this SNP results in aberrations in Siglec-8 structure and ligand binding, and subsequently leads to alterations in Siglec-8's function. As a result, we investigated the effect of this coding SNP on Siglec-8 ligand-binding activity using a synthetic polymer decorated with $6^{\prime}$-sulfo-sLe ${ }^{\mathrm{x}}$ when tested on HEK293 cells stably transfected with the rs 10409962 risk allele or the WT allele. We found no difference in binding to $6^{\prime}$-sulfo-sLe ${ }^{\mathrm{x}}$ ligand-containing polymers between transfectants with or without the risk allele, suggesting that this point mutation does not alter its ligand-binding activity. However, it is possible that the interaction between Siglec-8 and its natural ligand is different and not adequately reflected by the artificial polymer ligand. Alternatively, the assay used for testing interactions might not be sensitive enough to detect the potential differences between Siglec-8 molecules with $170 \mathrm{~S}$ versus 170P. However, this is the best way to currently explore this issue because the natural Siglec- 8 ligand remains to be determined. Another possibility is that it could alter the levels of Siglec-8 surface expression on eosinophils. Further studies are clearly needed to elucidate the mechanisms underlying the role of rs10409962 in the pathogenesis of asthma.

We recognized that, after Bonferroni Multiple testing correction, the SNP rs36498 was the only SNP significantly associated with asthma in the African American case-control group (adjusted $P=6.29 \times 10^{-4}$ ). Bonferroni correction offers the most conservative approach to control for false positives, and we therefore believe that the association with asthma for SNP rs36498 is more likely to be true among African Americans. Interestingly, the haplotype centered on rs36948 showed enhanced association with asthma and in particular, a haplotype (TA) carrying two promoter SNPs of Siglec-8 (rs36498 and rs11672925) demonstrated increased association when compared with either SNP individually. These findings suggest that SNP rs36498 and others in the promoter region of Siglec- 8 may be critical in conferring susceptibility to asthma. Hence, more SNPs with comprehensive coverage within the promoter region of Siglec- 8 are clearly needed to be genotyped, with the aim of identifying the variant(s) influencing transcriptional regulation. Also, we observed a significant association for SNP rs36498 and haplotypes carrying rs36948 among the Brazilian families, but the global $P$-value from the strongest haplotype is not as impressive as the single SNP rs36498. It can be speculated that the LD-genotyped SNPs are not great in coverage $(2.6 \mathrm{~kb} / \mathrm{SNP})$ and the signal flagged by the single promoter SNP is washed out by the SNPs surrounding it. An additional association was observed for SNP rs6509541 as an individual or in combination with other SNPs in the $3^{\prime}$-UTR and downstream from Siglec-8 and tIgE in the African American population, and replicated in the Brazilian population. This finding suggests a role for SNP rs6509541 via Siglec-8 in allergic diseases because tIgE is a useful marker for assessing allergic diseases. ${ }^{35}$

Given that Siglec-8 is a key molecule involved in facilitating eosinophil apoptosis and that mutations in Siglec- 8 may also be involved in other allergic and eosinophilic disorders, we investigated whether Siglec-8 SNPs are associated with other human eosinophilic disorders like EE. We selected eight SNPs encompassing the Siglec-8 gene with a coverage of $2 \mathrm{~kb} / \mathrm{SNP}$, including those associated with asthma. However, none of the SNPs were significantly associated with EE among the Caucasian population. Of these, four SNPs (rs10406692, rs3829659, rs39711, rs10420357) were further replicated among 68 EE families, but no significant association was observed (data not shown). The results suggest that Siglec- 8 does not associate with susceptibility to EE at least in Caucasians. However, further studies may be needed to get a comprehensive coverage of genetic variants in and around the Siglec- 8 gene in order to fully exclude Siglec- 8 as a candidate gene for EE.

In summary, we tested for associations between Siglec- 8 SNPs and asthma, $\operatorname{tgE}$, and $\mathrm{EE}$ in diverse populations. We are able, for the first time, to provide evidence of association between Siglec- 8 SNPs and asthma and tIgE in different racial populations. The strongest association was seen for SNP rs36498 in the promoter region of Siglec-8, even after correction for multiple tests, suggesting that the Siglec- 8 gene may be associated with susceptibility to asthma. The finding that Siglec-8 SNPs were not associated with EE suggests that the associa- 
tion is not simply due to atopy, which is found in similar percentages in these otherwise unrelated disorders. Further studies are needed to identify additional genetic variants, with a particular focus on the promoter region of the Siglec- 8 gene. As interaction assay failed to show any changes in ligand-binding activity on HEK293 cells stably transfected with the rs10409962 risk allele or the WT allele, additional studies are needed to determine exactly how this SNP might affect the biology of Siglec- 8 and contribute to the pathophysiology of asthma.

\section{CONFLICT OF INTEREST}

The authors declare no conflict of interest.

\section{ACKNOWLEDGEMENTS}

Funding source: This work was supported by a Dana Foundation Human Immunology Consortium grant; National Institutes of Health (NIH) grants HL087699, HL49612, AI50024, AI44840, HL075417, HL072433, AI72265, AI41040, ES09606, HL072433, and RR03048; and EPA grant 83213901; as well as by Glaxo-Smith-Kline (project WE445 entitled 'Immunogenetics of Schistosomiasis and Asthma'). KCB was supported in part by the Mary Beryl Patch Turnbull Scholar Program at Johns Hopkins. BSB was supported in part as a Cosner Scholar in Translational Research at Johns Hopkins. RAM was supported by the Intramural Research Program of the National Human Genome Research Institute, National Institutes of Health. LFZ was supported partially by the National Natural Science Foundation of China (30400191 and 30570797) and Jiangsu Key Principal Investigator of Medicine (RC2007043). We thank all the volunteers for generous participation in this study. We thank Deguang Mu of the Fourth Military Medical University, PR China. We thank Tracey Hand and Monica Campbell of the Johns Hopkins Bayview Genetics Research Facility, and Patricia Oldewurtel, Johns Hopkins University, for technical support. We thank Dr Bovin of the Russian Academy of Sciences, Moscow, Russia, for the polymer 6'-sulfo-sLex. We thank Dr Yukiko Maeda for help with genotyping of the Japanese sample.

1 von Gunten S, Bochner BS: Basic and clinical immunology of Siglecs. Ann N Y Acad Sci 2008; 1143: 61-82.

2 Kikly KK, Bochner BS, Freeman SD et al: Identification of SAF-2, a novel siglec expressed on eosinophils, mast cells, and basophils. J Allergy Clin Immunol 2000; 105: 1093-1100.

3 Bochner BS: Siglec-8 on human eosinophils and mast cells, and Siglec-F on murine eosinophils, are functionally related inhibitory receptors. Clin Exp Allergy 2009; 39 . 317-324.

4 Bochner BS, Alvarez RA, Mehta P et al: Glycan array screening reveals a candidate ligand for Siglec-8. J Biol Chem 2005; 280: 4307-4312.

5 Nutku E, Aizawa $\mathrm{H}$, Hudson SA, Bochner BS: Ligation of Siglec-8: a selective mechanism for induction of human eosinophil apoptosis. Blood 2003; 101: 5014-5020.

6 Nutku E, Hudson SA, Bochner BS: Mechanism of Siglec-8-induced human eosinophi apoptosis: role of caspases and mitochondrial injury. Biochem Biophys Res Commun 2005; 336: 918-924.

7 Nutku-Bilir E, Hudson SA, Bochner BS: Interleukin-5 priming of human eosinophils alters Siglec-8 mediated apoptosis pathways. Am J Respir Cell Mol Biol 2008; 38: $121-124$.

8 Zimmermann N, McBride ML, Yamada $Y$ et al: Siglec-F antibody administration to mice selectively reduces blood and tissue eosinophils. Allergy 2008; 63 1156-1163.

9 Song DJ, Cho JY, Miller M et al: Anti-Siglec-F antibody inhibits oral egg allergen induced intestinal eosinophilic inflammation in a mouse model. Clin Immunol 2009; 131: $157-169$
10 Zhang M, Angata T, Cho JY, Miller M, Broide DH, Varki A: Defining the in vivo function of Siglec-F, a CD33-related Siglec expressed on mouse eosinophils. Blood 2007; 109: 4280-4287.

11 Bochner BS: Verdict in the case of therapies versus eosinophils: the jury is still out. J Allergy Clin Immunol 2004; 113: 3-9.

12 Rothenberg ME, Hogan SP. The eosinophil. Annu Rev Immunol 2006; 24: 147-174.

13 Koh YI, Choi S: Blood eosinophil counts for the prediction of the severity of exerciseinduced bronchospasm in asthma. Respir Med 2002; 96: 120-125.

14 Jatakanon A, Lim S, Barnes PJ. Changes in sputum eosinophils predict loss of asthma control. Am J Respir Crit Care Med 2000; 161: 64-72.

15 Green RH, Brightling CE, McKenna S et al: Asthma exacerbations and sputum eosinophil counts: a randomised controlled trial. Lancet 2002; 360: 1715-1721.

16 Venanzi S, Malerba G, Galavotti R et al: Linkage to atopy on chromosome 19 in northeastern Italian families with allergic asthma. Clin Exp Allergy 2001; 31: 1220-1224.

17 Ober C, Cox NJ, Abney M et al: Genome-wide search for asthma susceptibility loci in a founder population. The Collaborative Study on the Genetics of Asthma. Hum Mol Genet 1998; 7: 1393-1398.

18 A genome-wide search for asthma susceptibility loci in ethnically diverse populations: The Collaborative Study on the Genetics of Asthma (CSGA). Nat Genet 1997; 15: 389-392.

19 Mathias RA, Grant AV, Rafaels $\mathrm{N}$ et al: A genome-wide association study on Africanancestry populations for asthma. J Allergy Clin Immunol 2009; e-pub ahead of print 10 November 2009

20 Standards for the diagnosis and care of patients with chronic obstructive pulmonary disease (COPD) and asthma. This official statement of the American Thoracic Society was adopted by the ATS Board of Directors, November 1986: Am Rev Respir Dis 1987; 136: 225-244.

21 Hamilton RG, Marcotte GV, Saini SS: Immunological methods for quantifying free and total serum IgE levels in allergy patients receiving omalizumab (Xolair) therapy. $\mathrm{J}$ Immunol Methods 2005; 303: 81-91.

22 Grant AV, Araujo MI, Ponte EV et al: High heritability but uncertain mode of inheritance for total serum IgE level and Schistosoma mansoni infection intensity in a schistosomiasis-endemic Brazilian population. J Infect Dis 2008; 198: 1227-1236.

23 Katz N, Chaves A, Pellegrino J: A simple device for quantitative stool thick-smear technique in Schistosomiasis mansoni. Rev Inst Med Trop Sao Paulo 1972; 14: 397-400.

24 Hattori T, Konno S, Hizawa N et al: Genetic variants in the mannose receptor gene (MRC1) are associated with asthma in two independent populations. Immunogenetics 2009; e-pub ahead of print 10 November 2009.

25 Blanchard C, Wang N, Stringer KF et al: Eotaxin-3 and a uniquely conserved geneexpression profile in eosinophilic esophagitis. J Clin Invest 2006; 116: 536-547.

26 Gao PS, Rafaels NM, Hand T et al: Filaggrin mutations that confer risk of atopic dermatitis confer greater risk for eczema herpeticum. J Allergy Clin Immunol 2009; 124: 507-513; 513 e501-507.

27 Maeda Y, Hizawa N, Takahashi D, Fukui Y, Konno S, Nishimura M: Genetic impact of functional single nucleotide polymorphisms in the $3^{\prime}$-UTR region of the chemoattractant receptor expressed on Th2 cells (CRTH2) gene on asthma and atopy in a Japanese population. Int Arch Allergy Immunol 2007; 142: 51-58.

28 Harris ML, Darrah E, Lam GK et al: Association of autoimmunity to peptidyl arginine deiminase type 4 with genotype and disease severity in rheumatoid arthritis. Arthritis Rheum 2008; 58: 1958-1967.

29 Hudson SA, Bovin NV, Schnaar RL, Crocker PR, Bochner BS: Eosinophil-selective binding and proapoptotic effect in vitro of a synthetic Siglec-8 ligand, polymeric 6'-sulfated sialyl Lewis x. J Pharmacol Exp Ther 2009; 330: 608-612.

30 Purcell S, Neale B, Todd-Brown $\mathrm{K}$ et al: PLINK: a tool set for whole-genome association and population-based linkage analyses. Am J Hum Genet 2007; 81: 559-575.

31 Horvath S, Xu X, Laird NM: The family based association test method: strategies for studying general genotype - phenotype associations. Eur J Hum Genet 2001; 9: 301-306.

32 Gauderman WJ: Sample size requirements for matched case-control studies of gene-environment interaction. Stat Med 2002; 21: 35-50.

33 Gabriel SB, Schaffner SF, Nguyen $\mathrm{H}$ et al: The structure of haplotype blocks in the human genome. Science 2002; 296: 2225-2229.

34 Blanchard C, Wang N, Rothenberg ME: Eosinophilic esophagitis: pathogenesis, genetics, and therapy. J Allergy Clin Immunol 2006; 118: 1054-1059.

35 Howell MD, Wollenberg A, Gallo RL et al: Cathelicidin deficiency predisposes to eczema herpeticum. J Allergy Clin Immunol 2006; 117: 836-841.

Supplementary Information accompanies the paper on European Journal of Human Genetics website (http://www.nature.com/ejhg) 